\title{
MRI findings in patients with a cervical spinal cord injury who do not show radiographic evidence of a fracture or dislocation
}

\author{
K Hayashi, K Yone, H Ito, M Yanase and T Sakou \\ Department of Orthopaedic Surgery, Faculty of Medicine, Kagoshima University, 8-31-1 Sakuragaoka, \\ Kagoshima City 891-01, Japan
}

\begin{abstract}
We investigated the usefulness of magnetic resonance imaging (MRI) in assessing cervical spinal cord injury in patients where there was no evidence of bone injury on radiographs, and examined the relationship between the MRI findings and the clinical prognosis of this injury. MRI allowed us to confirm directly the cause and severity of spinal cord compression in 30 of 31 cases. The patients with severe spinal cord compression demonstrated by MRI showed poor neurological improvement. In regard to the signal changes in the spinal cord, the patients who showed no signal change on T1- and T2-weighted images had a better prognosis. MRI is a very useful non-invasive adjunctive imaging modality for diagnosis of this injury and for the evaluation of the compressed spinal cord. It also demonstrates potential in predicting neurological recovery.
\end{abstract}

Keywords: cervical spinal cord injury; magnetic resonance imaging; prognosis

\section{Introduction}

Magnetic resonance imaging (MRI) has become widely used for assessing injury to the spinal cord. The relationship between the MRI findings and the prognosis has been reported by numerous investigators. ${ }^{1-4} \mathrm{We}$ recently investigated the usefulness of MRI in assessing cervical spinal cord injury in patients where there was no evident bone injury on radiographs, and examined the relationship between the MRI findings and the clinical prognosis of this injury.

\section{Methods}

The subjects were 31 patients with cervical spinal cord injury without bony injury evident on radiographs. There were 24 males and seven females, with ages ranging from 17 to 84 years (mean: 54.4 years). The observation period after injury ranged from 3 months to 2 years (mean: 13 months). The traumatic lesions of the spinal cord resulted in complete cord injury in one patient, the anterior cord syndrome in four, the central cord syndrome in 23 and the Brown-Séquard syndrome in three. The severity of the paralysis was evaluated according to the Frankel classification ${ }^{5}$ immediately after injury, at the time of the MRI and at the final observation. Operative treatment was performed in 17 patients and conservative treatment in 14 patients. In those who underwent operative treatment, MR studies were performed before surgery.

MRI studies were performed within 2 weeks after injury in 17 patients and after 2 weeks in 14 patients. Using 0.2-1.5 Tesla MRI devices, T1- and T2-weighted sagittal images were taken with the spin echo sequence.

On the MRI we observed the cause of spinal canal narrowing, the severity of spinal cord compression and the changes in signals in the spinal cord. The severity of cord compression was classified as mild (the narrowest diameter of the spinal cord $>2 / 3$ of the diameter at $\mathrm{C} 1$ level in each) and severe $(<2 / 3$ of the diameter at $\mathrm{C} 1 \mathrm{level})$. We examined the relationship between the MRI findings and the clinical symptoms.

\section{Results}

The severity of paralysis immediately after injury was A in one patient, $B$ in six, C in 14 and $D$ in 14 . Twenty-five of the 31 patients had paralysis at the final observation (Figure 1).

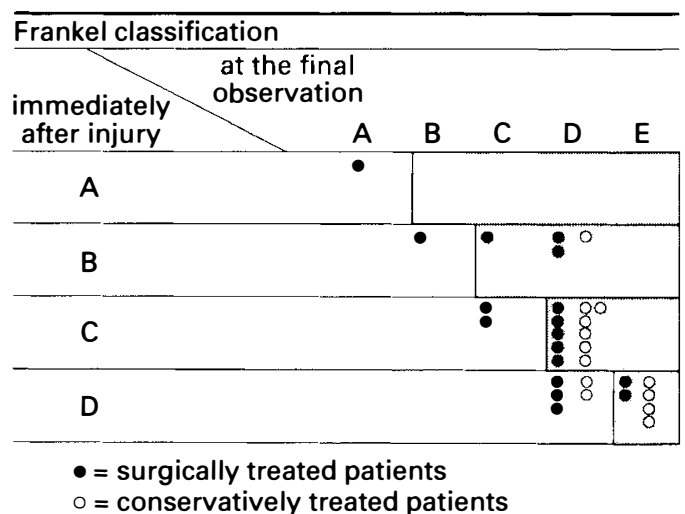

Figure 1 The severity of paralysis immediately after injury and at the final observation. The shaded area indicates neurological improvement according to the Frankel classification 
Spinal canal narrowing was caused by a herniated disc in seven patients, ossification of the posterior longitudinal ligament (OPLL) in eight patients and spondylosis in 15 patients. Spinal cord compression was absent in one patient.

Comparison of the severity of spinal cord compression with the prognosis of clinical symptoms

A number of patients with severe spinal cord compression as seen by MRI had severe paralysis immediately after the injury and showed poor neurological improvement at the final observation. Three of the 10 patients with severe spinal cord compression on the MRI who underwent operative treatment showed neurological improvement (Figure 2).

Comparison of the changes in spinal cord signals with the prognosis of clinical symptoms

Three types of MRI signal patterns were observed as follows: (1) no signal change in the spinal cord on T1and T2-weighted images, (2) no signal change on T1-weighted images but showed high signal intensity on T2-weighted images, (3) low signal intensity on T1weighted images and high signal intensity on T2weighted images. In the patients who showed no signal change in the spinal cord on T1- and T2-weighted images within 2 weeks after injury, the severity of paralysis was mild at the time of MRI and neurological symptoms improved markedly at the final observation (Figure 3). Of the patients who underwent MRI more than 2 weeks after injury, patients who showed no signal change in the spinal cord on T1- and T2-weighted images had mild paralysis at the time of MRI. None of the patients who showed low signal intensity on T1weighted images and high signal intensity on $\mathrm{T} 2$ weighted images showed any neurological improvement during the observation period (Figure 4).

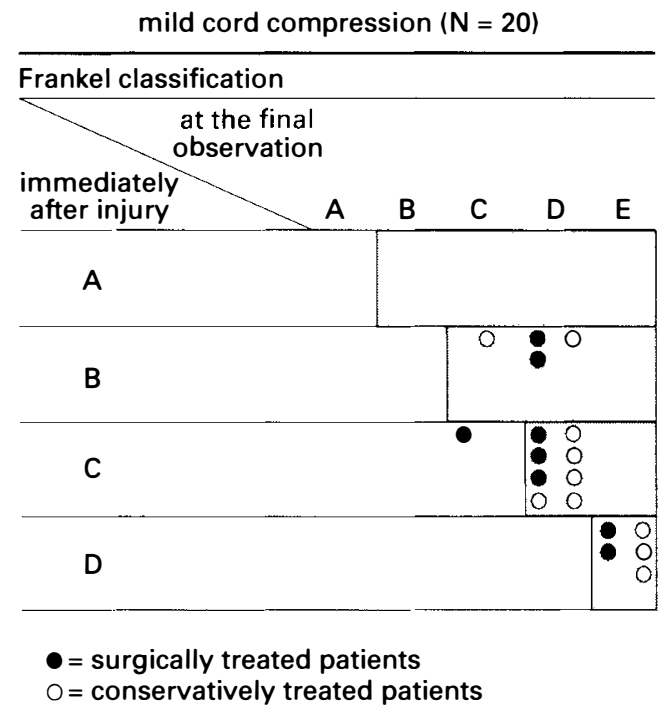

The cause of spinal canal narrowing

Comparison of the preoperative period with the prognosis of clinical symptoms

Operative treatment was performed within 2 weeks after injury in five cases and more than 2 weeks in 11 cases. Good clinical results were obtained in four of the five patients who were operated on within 2 weeks of injury and seven of the 11 patients who were operated on after more than 2 weeks. The differences of the clinical results between these two groups were not statistically significant by the $\chi^{2}$ test $(P=0.942)$.

\section{Case reports}

Case 1. A 40-year-old man

He was injured in a traffic accident, and had mild paralysis (Frankel D) at the time of MRI (3 days after the injury). The paralysis recovered completely at the final observation. On the MRI, the spinal cord was compressed mildly by a herniated disc and changes in signal intensity were not observed in the spinal cord (Figure 5).

\section{Case 2. A 72-year-old man}

He was injured by a fall, and had severe paralysis (Frankel B) at the time of MRI (7 days after the injury). The severe paralysis persisted during the observation period (Frankel C). On the MRI, the severe compression of the spinal cord caused by spondylotic changes and low signal intensity on T1-weighted images and high signal intensity on T2-weighted images were observed in the spinal cord (Figure 6).

\section{Discussion}

Bone injury was not evident in the radiographs in some patients who showed paralysis from a cervical cord injury. In such patients, invasive examinations such as myelography and CT myelography were required to make a diagnosis and assessment of injury to the cervical spinal cord. In our study, MRI allowed us to

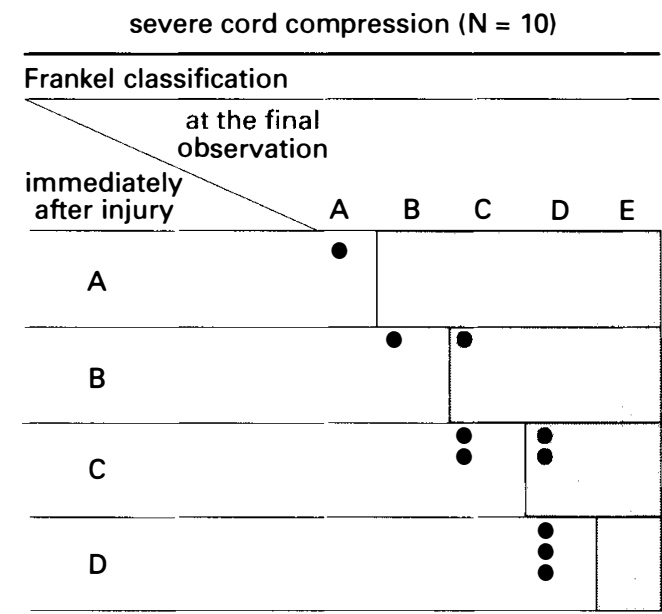

Figure 2 Comparison of the severity of spinal cord compression with the prognosis of clinical symptoms. The shaded area indicates neurological improvement according to the Frankel classification 

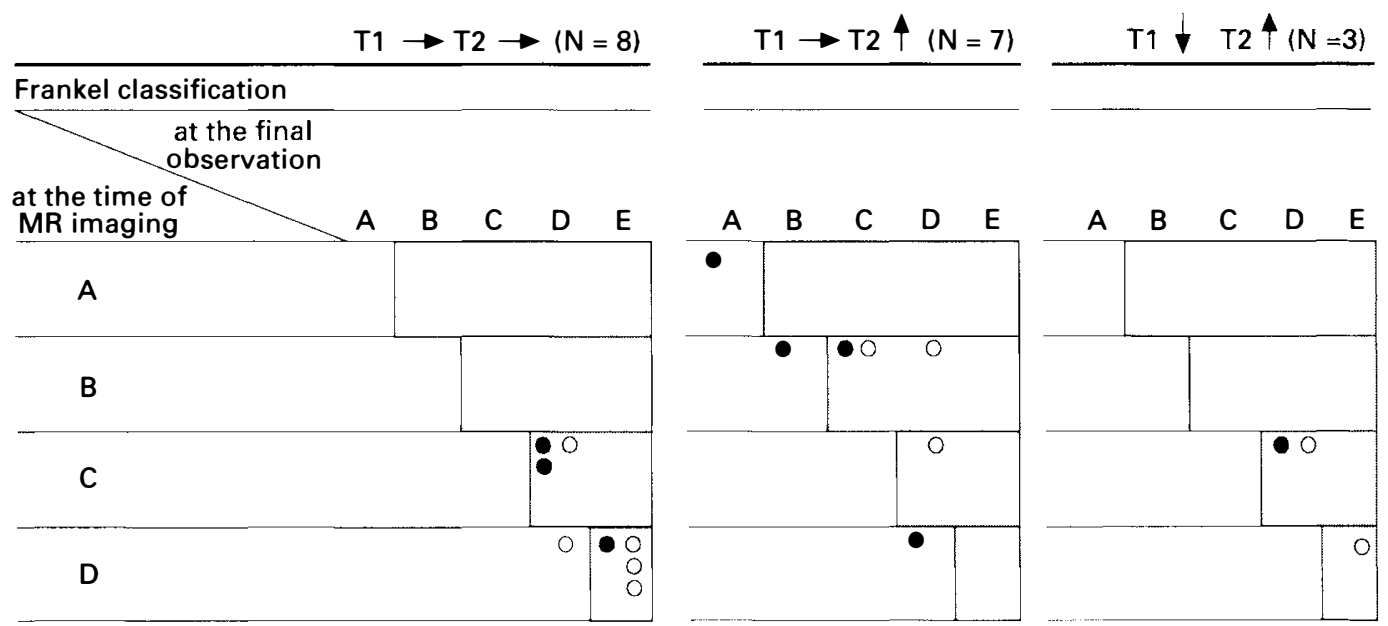

- surgically treated patients

$\mathrm{O}=$ conservatively treated patients

Figure 3 Comparison of changes in spinal cord signals with the prognosis of clinical symptoms for patients first imaged less than 2 weeks after injury. The shaded area indicates neurological improvement according to the Frankel classification

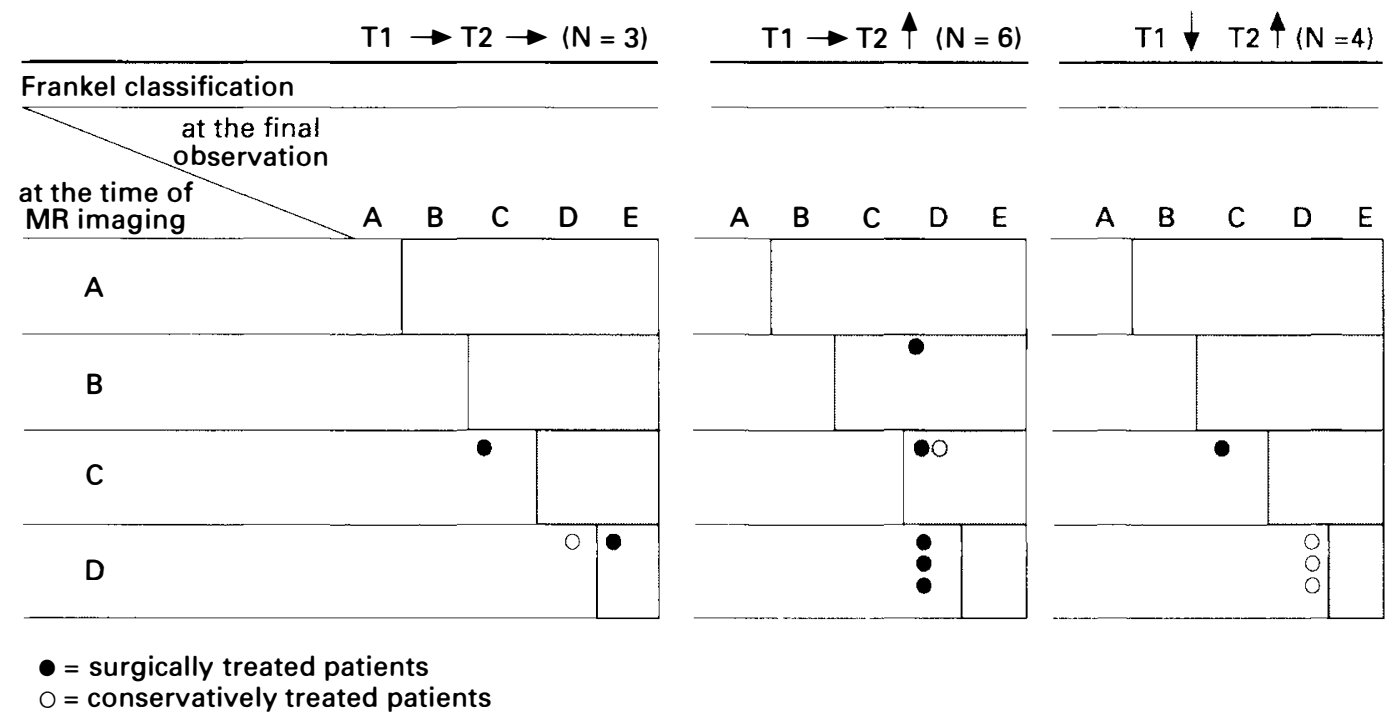

Figure 4 Comparison of signal changes in the spinal cord with the prognosis of clinical symptoms in the cases where MRI was performed within 2 weeks after injury. The shaded area indicates neurological improvement according to the Frankel classification. T1: T1-weighted images, T2: T2-weighted images, $\rightarrow$ : no signal changes, $\uparrow:$ high signal intensity, $\downarrow:$ low signal intensity

confirm directly the cause of spinal canal narrowing and the severity of spinal cord compression in 30 of 31 cases. Generally in the patients with this injury, the prognosis is considered favourable. But patients with severe spinal canal narrowing had poor neurological improvement. Three of the 10 patients with severe spinal cord compression on MRI who underwent operative treatment showed neurological improvement. We could not confirm the relationship between the preoperative period and the clinical results. In our opinion, although decompression is indicated for patients with severe spinal cord compression, we do not agree with the opinion that the operation should be performed as early as possible. MRI is a very useful non-invasive adjunctive imaging modality for the diagnosis of this injury and the evaluation of compression of the spinal cord.

In regard to signal changes in the spinal cord, patients who showed no signal change in the spinal cord on T1- and T2-weighted images had milder paralysis immediately after injury and had a better prognosis. Some investigators reported that a low signal intensity on T2-weighted images in the acute stage indicated bleeding within the spinal cord and hence a poor prognosis. . $^{1,3,4}$ In the present study, no such case was noted. This is probably because the magnitude of the 


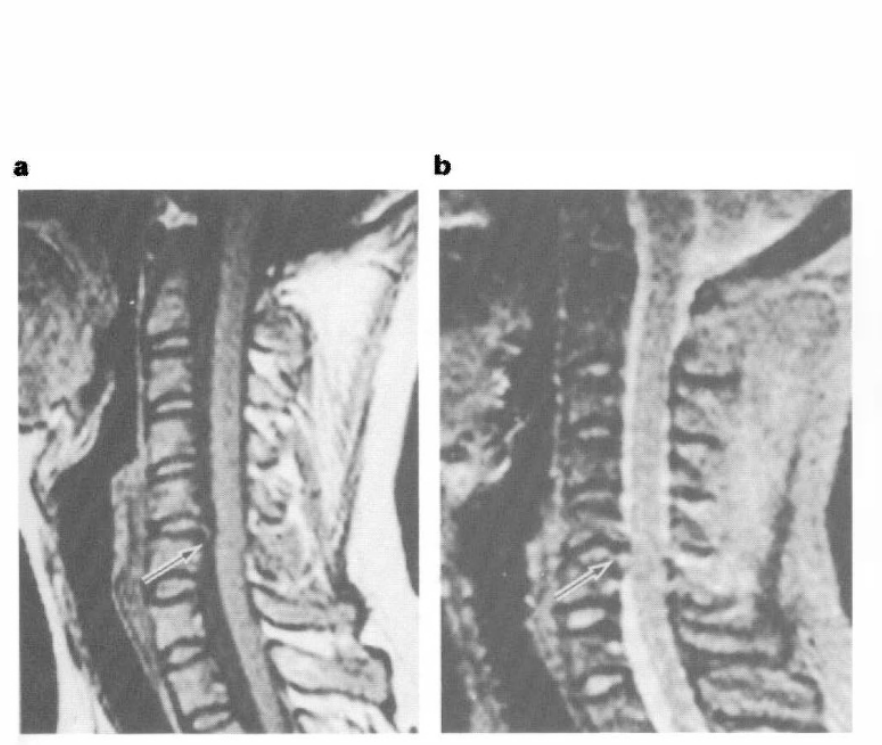

Figure 5 Case 1. MRI showed mildly compressed spinal cord by a herniated disc (arrows). No signal changes were observed either on T1-weighted (a) or on T2-weighted images (b)

extrinsic force in this injury was smaller than in the injury accompanied by fracture and dislocation. Absence of signal change on T1-weighted images was found. High signal intensity on T2-weighted images indicates oedema, and low signal intensity on T1weighted images and high signal intensity on $\mathrm{T} 2$ weighted images indicate necrosis in the acute stage. ${ }^{6}$ Though patients who showed low signal intensity on T1-weighted images and high signal intensity on T2weighted images have been thought to have a poor prognosis by some investigators, ${ }^{7}$ our results were not consistent with their results. This discrepancy may be attributable to involvement of the range of low signal intensity. In the chronic stage, low signal intensity on T1-weighted images and high signal intensity on T2weighted images pattern indicate cyst or syrinx. ${ }^{8}$ The prognosis seemed to be poor in cases with this pattern in the chronic stage.

\section{Conclusion}

Thirty-one patients with cervical spinal cord injury without evidence of bony injury on radiographs were observed with MRI. This is a very useful non-invasive adjunctive imaging modality for the diagnosis of this a

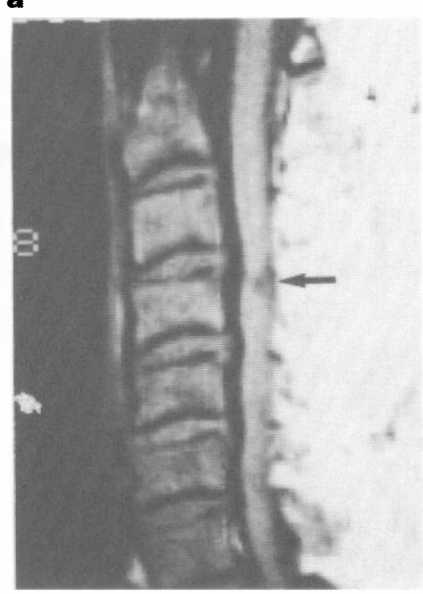

b

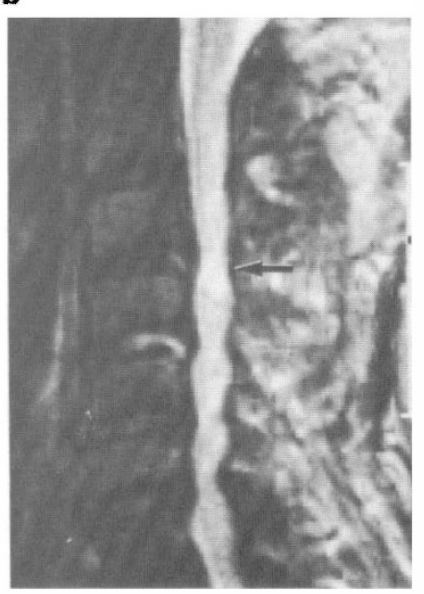

Figure 6 Case 2. MRI showed severe compression of the spinal cord caused by spondylotic changes. (a) Low signal intensity on T1-weighted image was observed (arrows). (b) High signal intensity on T2-weighted image was observed (arrows).

injury and the evaluation of the compressed spinal cord, and also demonstrated a potential in predicting the likelihood and degree of neurological recovery.

\section{References}

1 Hackney DB et al. Hemorrhage and edema in acute spinal cord compression: demonstration by MR imaging. Radiology 1986; 161: $387-390$.

2 Kulkarni MV et al. Acute spinal cord injury: MR imaging at 1.5T. Radiology 1987; 164: 837-843.

3 Flanders $\mathrm{AE}$ et al. Acute cervical spine trauma: Correlation of MR imaging findings with degree of neurologic deficit. Radiology 1990; 177: 25-33.

4 Marciello MA et al. Magnetic resonance imaging related to neurologic outcome in cervical spinal cord injury. Arch Phys Med Rehabil 1993; 74: 940-946.

5 Frankel HL et al. The value of postural reduction in the initial management of closed injuries of the spine with paraplegia and tetraplegia. Paraplegia 1969; 7: 179-192.

6 Fujii H, Yone K, Sakou T. Magnetic resonance imaging study of experimental acute spinal cord injury. Spine 1993; 18: 2030-2034.

7 Yamshita Y et al. Chronic injuries of the spinal cord: Assessment with MR imaging. Radiology 1990; 175: 849-854.

8 Enzmann DR, DeLaPaz RL, Rubin JB. Magnetic Resonance of the Spine. Mosby: St Louis, 1990, pp 237-259. 